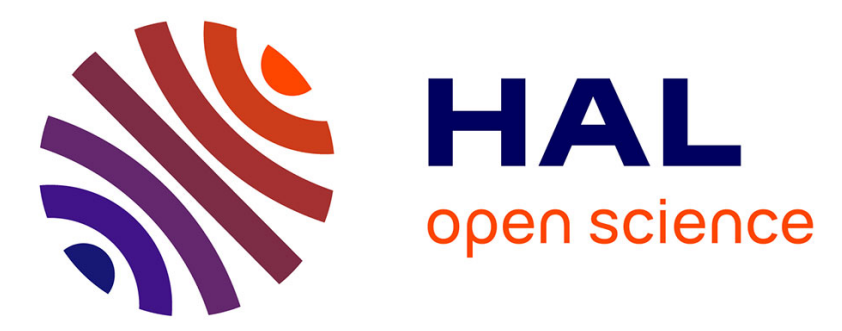

\title{
Application-Based Analysis of Transformations of Uncertain Dynamical Systems Into a Cooperative Form
}

\author{
Julia Kersten, Harald Aschemann, Andreas Rauh
}

\section{To cite this version:}

Julia Kersten, Harald Aschemann, Andreas Rauh. Application-Based Analysis of Transformations of Uncertain Dynamical Systems Into a Cooperative Form. 2021 25th International Conference on Methods and Models in Automation and Robotics (MMAR), Aug 2021, Międzyzdroje, Poland. pp.162167, 10.1109/MMAR49549.2021.9528439 . hal-03419427

\section{HAL Id: hal-03419427 \\ https://hal-ensta-bretagne.archives-ouvertes.fr/hal-03419427}

Submitted on 22 Nov 2021

HAL is a multi-disciplinary open access archive for the deposit and dissemination of scientific research documents, whether they are published or not. The documents may come from teaching and research institutions in France or abroad, or from public or private research centers.
L'archive ouverte pluridisciplinaire HAL, est destinée au dépôt et à la diffusion de documents scientifiques de niveau recherche, publiés ou non, émanant des établissements d'enseignement et de recherche français ou étrangers, des laboratoires publics ou privés. 


\section{Application-Based Analysis of Transformations of Uncertain Dynamical Systems Into a Cooperative Form}

\author{
Julia Kersten, Harald Aschemann \\ Chair of Mechatronics, University of Rostock \\ 18059 Rostock, Germany \\ Email: \{Julia.Kersten, Harald.Aschemann\}@uni-rostock.de
}

\author{
Andreas Rauh \\ ENSTA Bretagne, Lab-STICC \\ 29806 Brest, France \\ Email: Andreas.Rauh@interval-methods.de
}

\begin{abstract}
Uncertainty in dynamical system descriptions can have different sources. Whether it be mathematical model simplifications, manufacturing tolerances, and imperfect measurements resulting in parameter uncertainties or be it some kind of time-varying uncertain parameters as an interpretation of state dependencies in quasi-linear state-space representations. On the one hand, uncertainties can be represented as probability distributions in the stochastic case, which can be handled, for example by Monte-Carlo methods. However, those do not allow for the computation of worst-case bounds of the sets of reachable states. On the other hand, interval representations do allow this, which is why we will use those as a bounded error framework in the presented paper. When dealing with interval uncertainty, the rigorous computation of guaranteed state enclosures is a difficult task. Due to conservatism and/or the wrapping effect, overestimation is a common problem. The paper discusses not only a suitable control approach based on LMIs to stabilize an uncertain system but also a cooperativity-enforcing approach, which acts as a countermeasure to the wrapping effect. This simplifies the computation of guaranteed state enclosures in comparison with different methods to get the least conservative hull of the reachable state intervals. This is all done for a boom crane as a real-life application scenario.
\end{abstract}

\section{INTRODUCTION}

Generally, interval enclosures are computed with commonly used general-purpose, set-valued solvers for initial value problems (IVPs) for ordinary differential equations (ODEs), cf. [1], [2]. The computed state enclosures are often subject to the wrapping effect producing large overestimation, which leads to (interval) bounds that are much wider than the actually reachable sets of states. This effect typically occurs if complex shaped regions in a multi-dimensional state-space are replaced by simple shapes such as intervals [3] or zonotopes [4], cf. [5]. Although numerous approaches exist for a reduction of the wrapping effect, it cannot be eliminated fully in several practically relevant applications. Moreover, these contermeasures are typically quite computationally expensive and rarely allow for an application of these approaches in a real-time control or state estimation framework. In contrast to that, the property of cooperativity can simplify this to a large extent. Here, the solution of a single IVP with an interval box as initial states can be replaced by solving two decoupled IVPs for the infimum $\underline{\mathbf{x}}(t)$ and the supremum $\overline{\mathbf{x}}(t)$ with the corresponding initial states $\underline{\mathbf{x}}(0)=\inf \left\{\left[\mathbf{x}_{0}\right]\right\}$ as well as $\overline{\mathbf{x}}(0)=\sup \left\{\left[\mathbf{x}_{0}\right]\right\}$, respectively ${ }^{1}$. For this, we make use of the so-called positivity of a system model [6]

$$
\dot{\mathbf{x}}(t)=\mathbf{f}(\mathbf{x}(t)), \quad \mathbf{x} \in \mathbb{R}^{n},
$$

which is given when it is guaranteed that state trajectories $\mathbf{x}(t)$ starting in the positive orthant

$$
\mathbb{R}_{+}^{n}=\left\{\mathbf{x} \in \mathbb{R}^{n} \mid x_{i} \geq 0 \quad \forall i \in\{1, \ldots, n\}\right\}
$$

stay in this positive orthant for all $t \geq 0$ because $\dot{x}_{i}(t)=$ $f_{i}\left(x_{1}, \ldots, x_{i-1}, 0, x_{i+1}, \ldots x_{n}\right) \geq 0$ holds for all components $i \in\{1, \ldots, n\}$ of the state vector as soon as the state $x_{i}$ reaches the value $x_{i}=0$. This holds, when the following, sufficient criterion for cooperativity [7] of autonomous dynamic systems is fulfilled. If for a continuous-time dynamical system (1) all off-diagonal elements $J_{i, j}, i, j \in\{1, \ldots, n\}, i \neq j$, of the corresponding Jacobian

$$
\mathbf{J}=\frac{\partial \mathbf{f}(\mathbf{x})}{\partial \mathbf{x}}
$$

are strictly non-negative according to $J_{i, j} \geq 0, \quad i, j \in$ $\{1, \ldots, n\}, \quad i \neq j$, the system is cooperative and (3) is a Metzler matrix. In previous works, e.g. [8], [9], it was shown that there is a possibility to transform originally noncooperative systems into a cooperative form. Here, one needs to differentiate between systems with purely real eigenvalues and ones with conjugate-complex eigenvalue pairs. This means, that it is often easier to apply the transformation to a stabilized system and not to search for an eigenvalue placement by means of state feedback which enforces cooperativity as a much more restrictive task. As mentioned, transformed systems can be used to compute the guaranteed interval enclosures of all reachable states, which can than be used to optimize the controller gains for state-dependent uncertainties as shown in [10], further reducing the conservativity of the states in the $n$-dimensional interval box

$$
\mathbf{x}(t) \in[\mathbf{x}](t)=\left[\begin{array}{c}
{\left[\underline{x}_{1}(t) ; \bar{x}_{1}(t)\right]} \\
\vdots \\
{\left[\underline{x}_{n}(t) ; \bar{x}_{n}(t)\right]}
\end{array}\right]
$$

\footnotetext{
${ }^{1}$ For a fully verified solution of the IVPs, it is necessary to deal with bounds on the time discretization errors. For appropriate simulation procedures see [2]. However, in many control engineering applications, the use of fully verified solvers is often not necessary because uncertainty in the initial conditions is then much larger than the time discretization error of numerical solvers (e.g. Runge-Kutta methods) with sufficiently small integration step sizes.
} 
with $\underline{\mathbf{x}} \in \mathbb{R}_{+}^{n}$. Here, inf $\left(\left[x_{i}\right]\right)=\underline{x}_{i}$ represents the infimum and $\sup \left(\left[x_{i}\right]\right)=\bar{x}_{i}$ the supremum of each vector component $\left[x_{i}\right]=\left[\underline{x}_{i} ; \bar{x}_{i}\right], \underline{x}_{i} \leq x_{i} \leq \bar{x}_{i}, i \in\{1, \ldots, n\}$.

The unique contribution of this paper is the combination of a robust LMI-based control design and interval methods, which are applied to a boom crane. The general approach will be given in Sec. II, where we will first discuss the implementation of stabilizing controllers in greater detail, focusing on the general applicability of the system to different stability regions as well as further input optimizations by exploiting $\mathrm{H}_{2}$ or $H_{\infty}$-norms. Additionally, the transformation approach will be explained for systems with conjugate-complex eigenvalues in foresight of the application scenario at hand. This will be presented in Sec. III, where the oscillation of a load on a boom crane is attenuated. Sec. IV will focus on simulation results analyzing the influence of interval splitting methods to further reduce conservativity. Finally, a conclusion and an outlook are given in Sec. V.

\section{GENERAL APPROACH}

In the following, we assume a dynamical system to be uncertain due to a parameter uncertainty

$$
\dot{\mathbf{x}}=\mathbf{A}(\mathbf{p}) \cdot \mathbf{x}+\mathbf{B}(\mathbf{p}) \cdot \mathbf{u}
$$

and/or a state dependency resulting from a reformulation of a symbolic nonlinear system $\dot{\mathbf{x}}=\mathbf{f}(\mathbf{x}, \mathbf{u})$ into a quasi-linear state-space representation

$$
\dot{\mathbf{x}}=\mathbf{A}(\mathbf{x}) \cdot \mathbf{x}+\mathbf{B}(\mathbf{x}) \cdot \mathbf{u} .
$$

As stated in [8], the later on discussed transformation approach into a cooperative system model is chosen based on the eigenvalues. Here, we distinguish between systems with purely real and ones with conjugate complex eigenvalue pairs. As a controller influences the location of the eigenvalues, it is introduced before the transformation. Furthermore, the introduction of a state feedback controller

$$
\mathbf{u}=-\mathbf{K} \mathbf{x} \quad \text { or } \quad \mathbf{u}=-\mathbf{K}(\mathbf{x}) \cdot \mathbf{x},
$$

respectively, with vanishing feedforward signal reflects the structure of an autonomous system as stated in Sec. I, so that the system to be transformed becomes

$$
\dot{\mathbf{x}}=(\mathbf{A}(\mathbf{x})-\mathbf{B}(\mathbf{x}) \cdot \mathbf{K}(\mathbf{x})) \cdot \mathbf{x}=\mathbf{A}_{\mathrm{C}}(\mathbf{x}) \cdot \mathbf{x},
$$

where the controlled system matrix $\mathbf{A}_{\mathrm{C}}$ is then transformed into a Metzler structure. This section will give a general overview on both, the control and transformation approaches.

\section{A. LMI-based robust control with soft control effort limitation}

As published before, e.g. in [11], [12], LMI techniques are used again to achieve a robust stabilization of the system dynamics despite uncertain parameters and/or state dependencies of the matrices $\mathbf{A}$ and $\mathbf{B}$, respectively. For that, we overapproximate the quasi-linear system model (6) with a polytopic uncertainty representation, see [13]. Here, all uncertainties, parameter and state dependency, are summed up with parameter-dependent system and input matrices $\mathbf{A}(\mathbf{x}, \mathbf{p})$ and $\mathbf{B}(\mathbf{x}, \mathbf{p})$ or $\mathbf{A}(\mathbf{p}), \mathbf{B}(\mathbf{p})$, respectively, to achieve robustness of the control design. By that, the quasi-linear state-space representation is embedded into a polytopic uncertainty model in which uncertainty is accounted for by a convex combination of extremal system realizations. Here, the tight outer enclosure of all reachable states for which the following controller produces stable dynamic behavior is represented with $\mathrm{x} \in[\boldsymbol{\mathcal { X }}]$. This model can be described by the following convex combination of suitably chosen vertex matrices

$$
\begin{aligned}
\mathcal{D}=\{ & {[\mathbf{A}(\boldsymbol{\xi}), \mathbf{B}(\boldsymbol{\xi})] \mid[\mathbf{A}(\boldsymbol{\xi}), \mathbf{B}(\boldsymbol{\xi})] } \\
& \left.=\sum_{\nu=1}^{n_{\nu}} \xi_{v} \cdot\left[\mathbf{A}_{\nu}, \mathbf{B}_{\nu}\right] ; \sum_{\nu=1}^{n_{\nu}} \xi_{\nu}=1 ; \xi_{\nu} \geq 0\right\}
\end{aligned}
$$

with the help of the vector $\boldsymbol{\xi}=\left[\begin{array}{lll}\xi_{1} & \ldots & \xi_{\nu}\end{array}\right]^{T}$.

In (9), each of the vertex matrices $\mathbf{A}_{\nu}=\mathbf{A}_{\nu}(\mathbf{p})$ and $\mathbf{B}_{\nu}=\mathbf{B}_{\nu}(\mathbf{p})$ depends on the vector of independent parameters $\mathbf{p} \in \mathbb{R}^{n_{\mathrm{p}}}$ in an affine manner. All independent parameters are contained in the interval box $[\mathbf{p}]=[\mathbf{p} ; \overline{\mathbf{p}}]$ with the component-wise defined bounds $p_{i} \leq p_{i} \leq \bar{p}_{i}$, $i \in\left\{1, \ldots, n_{\mathrm{p}}\right\}$. The evaluation of $\mathbf{A}(\mathbf{p})$ and $\mathbf{B}(\mathbf{p})$ is performed for each of the vertices

$\mathcal{P}=\left\{\left[\begin{array}{c}\underline{p}_{1} \\ \underline{p}_{2} \\ \vdots \\ \underline{p}_{n_{\mathrm{p}}}\end{array}\right],\left[\begin{array}{c}\bar{p}_{1} \\ \underline{p}_{2} \\ \vdots \\ \underline{p}_{n_{\mathrm{p}}}\end{array}\right], \ldots,\left[\begin{array}{c}\bar{p}_{1} \\ \bar{p}_{2} \\ \vdots \\ \bar{p}_{n_{\mathrm{p}}}\end{array}\right]\right\}=\left\{\mathbf{p}^{\langle 1\rangle}, \ldots, \mathbf{p}^{\left\langle n_{\nu}\right\rangle}\right\}$

resulting in $n_{\nu}=2^{n_{\mathrm{p}}}$ extremal models that need to be taken into consideration for the robust control design under the assumption of mutually independent parameters. With the help of so-called $\Gamma$-regions, feasible regions of eigenvalues of the closed-loop control system are defined and, hence, the desired performance is adapted to the user's special requirements. With the Laplace variable $s \in \mathbb{C}$, these regions are defined by domains of strict negative definiteness of the matrix inequality

$$
\mathbf{F}_{\Gamma}(s)=\mathbf{D}_{0}+s \mathbf{D}_{1}+\bar{s} \mathbf{D}_{1}^{T} \prec 0
$$

that needs to be satisfied for all eigenvalues of the closedloop system with $\bar{s}$ denoting the conjugate complex of the Laplace variable $s$. Here, the real-valued parameter matrices $\mathbf{D}_{0}=\mathbf{D}_{0}^{T}$ and $\mathbf{D}_{1}$ provide flexible possibilities to define $\Gamma$-stability regions such as ellipses, hyperbolas, parabolas, cones, and strips in the complex plane [14]. The inequality $\mathbf{F}_{\Gamma} \prec 0$ is reformulated into an equivalent LMI to derive a computationally tractable solution approach. If all eigenvalues of a real-valued system matrix $\mathbf{A}$ lie within the interior of the region (11), a positive definite matrix $\mathbf{P}=\mathbf{P}^{T} \succ 0$ exists that fulfills the matrix inequality [13]

$$
\mathbf{D}_{0} \otimes \mathbf{P}+\mathbf{D}_{1} \otimes(\mathbf{A P})+\mathbf{D}_{1}^{T} \otimes(\mathbf{A P})^{T} \prec 0 .
$$

In (12), the matrix $\mathbf{P}$ defines a Lyapunov function $V(\mathbf{x})=$ $\frac{1}{2} \mathbf{x}^{T} \mathbf{P} \mathbf{x}>0$ for $\mathbf{x} \neq \mathbf{x}_{\mathrm{s}}=\mathbf{0}$ with which stability of the dynamic system $\dot{\mathbf{x}}=\mathbf{A x}$ can be proven. Moreover, as mentioned before, $\mathbf{D}_{0}=\mathbf{D}_{0}^{T}$ and $\mathbf{D}_{1}$ can be specified to the user's needs. Considering, e.g. the absolute stability margin $\gamma>0$ as the design goal $\Re\{s\}<-\gamma$ according to $F_{\Gamma}=2 \gamma+s+\bar{s} \prec 0$, corresponds to the setting $D_{0}=2 \gamma$ and $D_{1}=1$. Note that pure Hurwitz stability is trivially included in this formulation by choosing $\gamma=0$. To use the inequality (12) for control design, it is reformulated into an LMI according to

$\mathbf{D}_{0} \otimes \mathbf{Q}+\mathbf{D}_{1} \otimes\left(\mathbf{Q A}_{\nu}^{T}-\mathbf{Y}^{T} \mathbf{B}_{\nu}^{T}\right)+\mathbf{D}_{1}^{T} \otimes\left(\mathbf{A}_{\nu} \mathbf{Q}-\mathbf{B}_{\nu} \mathbf{Y}\right) \prec 0$ 
after a linearizing change of variables $\mathbf{Q}=\mathbf{P}^{-1}$ and $\mathbf{K}=\mathbf{Y} \mathbf{P}$. Note that, also optimality criteria such as robust $H_{2}$ and $H_{\infty}$ tasks can be taken into account by the same LMI-based design framework. For the given scenario, an $\mathrm{H}_{2}$-norm optimization, see [13], as a soft limitation to the control effort has been performed, adding

$$
\left[\begin{array}{cc}
\mathbf{Q} \mathbf{A}_{\nu}^{T}-\mathbf{Y}^{T} \mathbf{B}_{\nu}^{T}+\mathbf{A}_{\nu} \mathbf{Q}-\mathbf{B}_{\nu} \mathbf{Y} & \mathbf{0} \\
\mathbf{0} & -\mathbf{I}
\end{array}\right] \prec 0
$$

(without models for process noise) and

$$
\left[\begin{array}{cc}
\mathbf{Q} & \mathbf{Q C} \mathbf{C}_{2}^{T}-\mathbf{Y}^{T} \mathbf{D}_{22}^{T} \\
\mathbf{C}_{2} \mathbf{Q}-\mathbf{D}_{22} \mathbf{Y} & \mathbf{Z}
\end{array}\right] \succ 0
$$

as two additional LMIs to be taken into account together with Eq. (13). Furthermore, a cost function containing $\min \{\operatorname{trace}\{\mathbf{Z}\}\}$ is introduced. This corresponds to a robust linear quadratic controller approach, where

$$
\mathbf{y}_{2}=\underbrace{\left[\begin{array}{c}
\mathbf{0} \\
\mathcal{Q}^{\frac{1}{2}}
\end{array}\right]}_{\mathbf{C}_{2}} \mathbf{x}(t)+\underbrace{\left[\begin{array}{c}
\mathcal{R}^{\frac{1}{2}} \\
\mathbf{0}
\end{array}\right]}_{\mathbf{D}_{22}} \mathbf{u}(t)
$$

from $J=\frac{1}{2} \int_{0}^{\infty}\left(\mathbf{x}^{T} \mathcal{Q} \mathbf{x}+\mathbf{u}^{T} \mathcal{R} \mathbf{u}\right) \mathrm{d} t$ holds, see [14]. With suitably chosen weighting matrices $\mathcal{Q}$ and $\mathcal{R}$, a compromise between the control effort and the integral of the quadratic control error can be made. Note that in all LMIs (13)-(15) the use of the index $\nu, \nu \in\left\{1, \ldots, 2^{n_{\mathrm{p}}}\right\}$, visualizes that robust stability for the uncertainty representation (9)-(10) with eigenvalues that are compatible with the domain $\mathbf{F}_{\Gamma} \prec 0$ defined in (11) is achieved as soon as a joint solution $\mathbf{Q} \succ 0$, $\mathbf{Y}$ of the LMIs (13)-(15) has been found that is valid for each of the vertices. This problem can be solved numerically by using commonly known LMI toolboxes like SEDUMI [15] in combination with YALMIP [16].

\section{B. Transformation of non-cooperative systems with conjugate- complex eigenvalues}

Implementing the controller designed in the previous subsection for the system following Eq. (8), we can assume a stabilized system with the controlled system matrix $\mathbf{A}_{\mathrm{C}}(\mathbf{x}, \mathbf{p})$. Now, as previously discussed, we need the domain for all reachable states $[\mathbf{x}](t) \subseteq[\mathcal{X}]$ for the complete operating horizon $t \in\left[t_{0} ; t_{\mathrm{f}}\right]$ to implement optimized controllers as shown in [11]. Knowing the guaranteed interval enclosures of reachable states is furthermore a question of safety to some systems. The property of cooperativity was found very useful for that, see [8]. Here, an approach was presented using a transformation of the system into a cooperative form. The computation of the worst-case bounds $\mathbf{x}(t) \in[\mathbf{v}(t) ; \mathbf{w}(t)]$ was simplified by solving IVPs independently for two decoupled bracketing systems [7] according to

$$
\begin{aligned}
\inf \left(\mathbf{A}_{\mathrm{C}}([\boldsymbol{\mathcal { X }}], \mathbf{p})\right) \cdot \mathbf{v}(t) & =\dot{\mathbf{v}}(t) \\
\leq \dot{\mathbf{x}}(t) \leq \mathbf{\mathbf { w }}(t) & =\sup \left(\mathbf{A}_{\mathrm{C}}([\mathcal{\mathcal { X }}], \mathbf{p})\right) \cdot \mathbf{w}(t)
\end{aligned}
$$

This assumes that $\mathbf{v} \in \mathbb{R}_{+}^{n}$ holds, otherwise, the reader ist referred to [17]. In our paper, we will restrict ourselves to the transformation approach for conjugate-complex eigenvalues in foresight to the application scenario at hand. This approach holds if the interval evaluation $\mathbf{A}_{\mathrm{C}}([\mathcal{X}], \mathbf{p})$ of the closedloop system matrix has conjugate-complex eigenvalue pairs.
For such systems, generally only time-varying transformations into the form (17) are possible, see [9], [18]. In these systems, the uncertainty is mapped into the locations of the eigenvalues themselves. This leads to a variability of the real and imaginary parts of conjugate-complex eigenvalues if the system matrices are evaluated for the whole range of uncertain parameters (here including state dependencies), see Fig. 1 as an illustrative example for a system of order $n=2$.

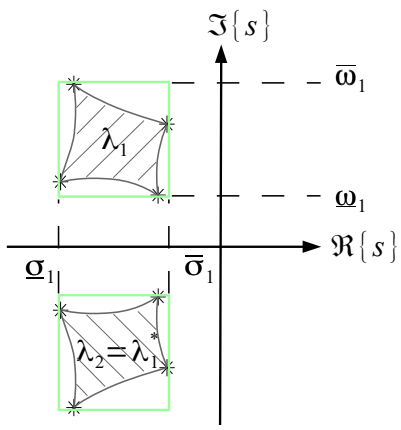

Figure 1. Possible locations of uncertain conjugate-complex eigenvalues.

For disjoint eigenvalues, the position of the worst-case eigenvalues $i \in\{1, \ldots, n\}$ for all possible vertex matrices, which were used before for a polytopic description of the uncertain system matrices, is marked by asterisk symbols. The axes-parallel boxes, defined by those positions, are used as a convex outer interval hull describing the extremal real and imaginary parts $\left[\sigma_{i}\right]=\left[\underline{\sigma}_{i} ; \bar{\sigma}_{i}\right]$ and $\left[\omega_{i}\right]=\left[\underline{\omega}_{i} ; \bar{\omega}_{i}\right]$ of a conjugate-complex eigenvalue pair. This approach is equally applicable to solve problems with purely real eigenvalues, and as in the case of the presented application scenario, a mixture of both. In the following, we will, hence, assume that the list of eigenvalues contains $\tilde{n}<\frac{n}{2}$ complex pairs, leading to $n^{*}=n-2 \tilde{n} \geq 0$ real eigenvalues, which are sorted in such a way that all complex pairs are listed first, see also [19]. Now, a first transformation into a block diagonal structure

$$
\tilde{\mathbf{A}}=\operatorname{blkdiag}\left(\tilde{\mathbf{A}}_{1}, \ldots, \tilde{\mathbf{A}}_{\tilde{n}+n^{*}}\right),
$$

is performed, where a pair of conjugate-complex eigenvalues with $\omega_{i}=-\omega_{i+1}, i \in\{1,3, \ldots, 2 \tilde{n}-1\}$, leads to a block entry

$$
\tilde{\mathbf{A}}_{j} \in\left[\begin{array}{cc}
{\left[\sigma_{j}\right]} & {\left[\omega_{j}\right]} \\
-\left[\omega_{j}\right] & {\left[\sigma_{j}\right]}
\end{array}\right], \quad j \in\{1,2, \ldots, \tilde{n}\},
$$

while an uncertain real eigenvalue $(i \in\{2 \tilde{n}+1,2 \tilde{n}+2, \ldots, n\})$ is reflected by $\tilde{\mathbf{A}}_{j} \in\left[\sigma_{i}\right], j=i-\tilde{n}$. For mutually disjoint eigenvalue enclosures, the structure of (18) is obtained with the transformation matrix

$$
\begin{gathered}
\tilde{\mathbf{T}}=\left[\tilde{\mathbf{T}}_{1}, \ldots, \tilde{\mathbf{T}}_{\tilde{n}+n^{*}}\right] \quad \text { with } \\
\tilde{\mathbf{T}}_{j} \in\left[\Re\left\{\left[\mathbf{v}_{j}\right]\right\}, \Im\left\{\left[\mathbf{v}_{j}\right]\right\}\right], \quad j \in\{1,2, \ldots, \tilde{n}\},
\end{gathered}
$$

corresponding to (19) and $\tilde{\mathbf{T}}_{j}=\left[\mathbf{v}_{j}\right], j=i-\tilde{n}$, for the real eigenvalues $i \in\{2 \tilde{n}+1,2 \tilde{n}+2, \ldots, n\}$. The time-varying transformation is performed by the matrix

$$
\mathbf{S}(t)=\operatorname{blkdiag}\left(\mathbf{S}_{1}(t), \ldots, \mathbf{S}_{\tilde{n}+n^{*}}(t)\right)=\left(\mathbf{S}^{-1}(t)\right)^{T}
$$

with the orthogonal blocks

$$
\mathbf{S}_{j} \in\left[\begin{array}{cc}
\cos \left(\left[\omega_{j}\right] t\right) & \sin \left(\left[\omega_{j}\right] t\right) \\
-\sin \left(\left[\omega_{j}\right] t\right) & \cos \left(\left[\omega_{j}\right] t\right)
\end{array}\right], j \in\{1,2, \ldots, \tilde{n}\},
$$


and

$$
\mathbf{S}_{\tilde{n}+1}=\ldots=\mathbf{S}_{\tilde{n}+n^{*}}=1 .
$$

The state-sapce representation of the related differential equation is calculated by

$$
\begin{aligned}
& \dot{\mathbf{z}}=\mathbf{T}^{-1}(t) \cdot\left(-\dot{\mathbf{T}}(t)+\mathbf{A}_{\mathrm{C}}([\mathcal{X}], \mathbf{p}) \cdot \mathbf{T}(t)\right) \cdot \mathbf{z} \\
& =\left((\dot{\mathbf{T}}(t))^{-1}+\mathbf{T}^{-1}(t) \cdot \mathbf{A}_{\mathrm{C}}([\mathcal{X}], \mathbf{p})\right) \cdot \mathbf{T}(t) \cdot \mathbf{z} \\
& \text { with } \quad \mathbf{x}(t) \in \mathbf{T}(t) \cdot[\mathbf{z}](t)=(\tilde{\mathbf{T}} \cdot \mathbf{S}(t)) \cdot[\mathbf{z}](t) .
\end{aligned}
$$

By symbolic simplification, before substituting interval variables into the transformation matrix $\mathbf{T}(t)$, it can be shown that $\dot{\mathbf{z}} \in[\mathbf{N}] \cdot[\mathbf{z}]$ holds, with $\mathbf{I}=\left[\begin{array}{ll}1 & 0 \\ 0 & 1\end{array}\right]$ and

$$
[\mathbf{N}]=\operatorname{blkdiag}\left(\left[\sigma_{1}\right] \cdot \mathbf{I}, \ldots,\left[\sigma_{\tilde{n}}\right] \cdot \mathbf{I},\left[\sigma_{\tilde{n}+1}\right], \ldots,\left[\sigma_{\tilde{n}+n^{*}}\right]\right) \text {. }
$$

Note that this simplification only holds if all imaginary parts $\omega_{j}$ are constant for known time intervals used in the simulation. Hence, for fast changes of $\omega_{j}(t)$ resulting from fast, arbitrary variations of $[\mathbf{x}](t) \subseteq[\mathcal{X}]$ as well as $\mathbf{p}$, Eq. (25) must be treated with care. Here, due to the decoupled diagonal structure independent of the sign of $\mathbf{z}$, the intervals can be calculated directly with

$$
\begin{aligned}
& {[\mathbf{z}]=\operatorname{blkdiag}\left(\left[e^{\left[\sigma_{1}\right] t} \cdot \mathbf{I}, \ldots, e^{\left[\sigma_{\tilde{n}}\right] t} \cdot \mathbf{I},\right.\right.} \\
&\left.\left.e^{\left[\sigma_{\tilde{n}+1}\right] t}, \ldots, e^{\left[\sigma_{\tilde{n}+n^{*}}\right] t}\right]\right) \cdot[\mathbf{z}](0) .
\end{aligned}
$$

This would not be possible, if the matrices in (17) had not this special form. For the numerical evaluation of the IVP corresponding to (25), the diagonal elements of (27) are replaced by the lower and upper interval bounds to obtain the bounding systems. Since $\mathbf{N}$ is evaluated for the eigenvalues, Hurwitz stability is verified for $\bar{\sigma}_{j}<0$. Extrema of the conjugate-complex eigenvalues are obtained by building the hull over their real and imaginary parts

$$
\begin{aligned}
& {\left[\sigma_{j}\right]=\left[\min \left(\sigma_{j}\right) ; \quad \max \left(\sigma_{j}\right)\right],} \\
& {\left[\omega_{j}\right]=\left[\min \left(\omega_{j}\right) ; \quad \max \left(\omega_{j}\right)\right] .}
\end{aligned}
$$

To determine $\tilde{\mathbf{T}}$ from (20), the hull over all eigenvectors needs to be found. Unfortunately, the evaluation of the eigenvectors for the vertices of a polytopic system model does not lead to the extremal values of the corresponding vector components from which a convex interval hull can be formed. Hence, interval eigenvectors are calculated using the function verifyeig of INTLAB [20] after subdividing the interval parameter domains of $\left[\mathbf{A}_{C}\right]$ and then normalizing to length 1 (using the INTLAB routine norm). In general, small subintervals are chosen as a countermeasure against overestimation in the eigenvalue and eigenvector computation. Since the axis-parallel box over the $n_{p}$ vertex matrices would be too conservative, we make use of subdividing the parameters for smaller subintervals $\left[p^{\kappa}\right] \subseteq[p]$ with $\kappa=1 \ldots L$ and then using the hull over all partial intervals. The hull over all subintervals denotes the interval eigenvalues and -vectors, respectively. Now, there are three possibilities to calculate a single initial state interval enclosure accounting for these subintervals for the transformed set of ODEs (25):

1) Using the hull over all eigenvectors according to Eq. (20) resulting in the transformation matrix

$$
\tilde{\mathbf{T}}=\left[\tilde{\mathbf{T}}_{1}, \ldots, \tilde{\mathbf{T}}_{\tilde{n}}\right] \quad \text { with }
$$

$$
\tilde{\mathbf{T}}_{j} \in\left[\bigcup_{\kappa=1}^{L}\left[\Re\left\{\left[\mathbf{v}_{j}\right]\right\}^{\kappa}\right], \bigcup_{\kappa=1}^{L}\left[\Im\left\{\left[\mathbf{v}_{j}\right]\right\}^{\kappa}\right]\right]
$$

and finally $[\mathbf{z}]=[\mathbf{T}]^{-1} \cdot[\mathbf{x}]$ with $[\mathbf{T}]=[\tilde{\mathbf{T}}] \cdot \mathbf{S}(t)$.

2) Using the hull over all transformation matrices

$$
[\tilde{\mathbf{T}}]=\bigcup_{\kappa=1}^{L}\left[\tilde{\mathbf{T}}^{\kappa}\right]
$$

and finally $[\mathbf{z}]=[\mathbf{T}]^{-1} \cdot[\mathbf{x}]$ with $[\mathbf{T}]=[\tilde{\mathbf{T}}] \cdot \mathbf{S}(t)$.

3) Calculating the hull over all initial state intervals

$$
[\mathbf{z}]=\bigcup_{\kappa=1}^{L}\left[\mathbf{z}^{\kappa}\right] \quad \text { with } \quad\left[\mathbf{z}^{\kappa}\right]=\left[\mathbf{T}^{\kappa}\right]^{-1} \cdot[\mathbf{x}]
$$

\section{APPLICATION SCENARIO}

The application scenario at hand is given by the task of oscillation attenuation for a boom crane with in-plane payload motion. The system is presented schematically in Fig. 2. Here,

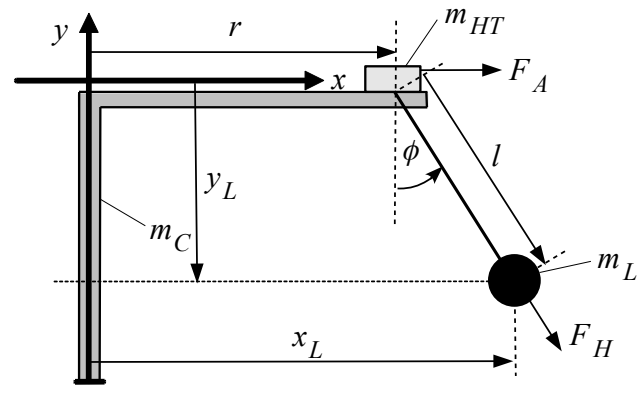

Figure 2. Schematic representation of a boom crane, generalized coordinates are the position $r$ and the angle $\phi$.

a rope with the length $l$ connects the mass of a payload $m_{L}$ to a moving carriage $m_{H T}$. Since the rope length is variable, it is chosen as an uncertain parameter. For a derivation of the equations of motion using Lagrange's equations of second kind

$$
\frac{\mathrm{d}}{\mathrm{d} t}\left(\frac{\partial L}{\partial \dot{\mathbf{q}}(t)}\right)-\frac{\partial L}{\partial \mathbf{q}(t)}+\frac{\partial D}{\partial \dot{\mathbf{q}}}=\mathbf{Q},
$$

the Lagrange function is defined by the difference of kinetic and potential energy $L=E_{\text {kin }}-E_{\text {pot }}$. Here, the overall kinetic energy of the system is

$$
\begin{aligned}
E_{\mathrm{kin}} & =E_{\mathrm{kin}, H T}+E_{\mathrm{kin}, L} \\
& =\frac{1}{2} m_{H T} \dot{r}^{2}(t)+\frac{1}{2} m_{L} \\
& \cdot\left[(\dot{r}(t)+l \cos (\phi(t)) \dot{\phi}(t))^{2}+(l \sin (\phi(t)) \dot{\phi}(t))^{2}\right],
\end{aligned}
$$

while the potential energy is described by

$$
E_{\text {pot }}=E_{\text {pot }, L}=m_{L} l g \cos (\phi(t)) .
$$

To evaluate Eq. (34), the vector of external forces $\mathbf{Q}=$ $\left[\begin{array}{ll}F_{A} & 0\end{array}\right]^{T}$ is given, where $F_{A}$ is the force acting on the carriage. Furthermore, a Rayleigh dissipation function with the pseudo energy $D=\frac{1}{2} d \dot{\phi}^{2}$ is introduced to account for internal velocity-proportional dissipation of the payload energy. With

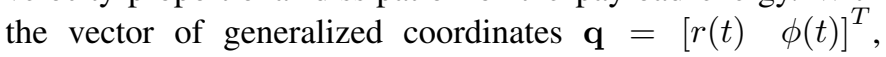


Lagrange's equation (34) is evaluated resulting in the two equations of motion

$$
\begin{gathered}
\left(m_{H T}+m_{L}\right) \ddot{r}+m_{L} l \cos (\phi) \ddot{\phi}-m_{L} l \sin (\phi) \dot{\phi}^{2}=F_{A} \\
m_{L} l \cos (\phi) \ddot{r}+m_{L} l^{2} \ddot{\phi}+m_{L} l g \sin (\phi)+d \dot{\phi}=0 .
\end{gathered}
$$

Assuming an underlying velocity control for the carriage, Eq. (37) can be replaced by

$$
T_{1} \ddot{r}(t)+\dot{r}(t)=v(t) .
$$

In the following, time arguments are omitted for the sake of compactness. When the state vector consists of the vector of generalized coordinates and their respective velocities $\mathbf{x}=\left[\begin{array}{ll}\mathbf{q}^{T} & \dot{\mathbf{q}}^{T}\end{array}\right]^{T}$, the resulting state-space representation is

$$
\dot{\mathbf{x}}=\left[\begin{array}{cccc}
0 & 0 & 1 & 0 \\
0 & 0 & 0 & 1 \\
0 & 0 & -\frac{1}{T_{1}} & 0 \\
0 & -\frac{g \cdot \operatorname{si}(\phi)}{l} & \frac{\cos (\phi)}{T_{1} l} & -d
\end{array}\right] \mathbf{x}+\left[\begin{array}{c}
0 \\
0 \\
\frac{1}{T_{1}} \\
-\frac{\cos (\phi)}{T_{1} l}
\end{array}\right] u
$$

where the input $u:=u(t)$ is given by the desired carriage velocity $v:=v(t)$. Here, the parameters $l$ and $d$ are uncertain, while the state-dependency of $\phi$ is treated as an intervalbounded and within its bounds time-varying uncertainty. To account for parameter dependency of the uncertain entries in $\mathbf{A}(\mathbf{x})$ and $\mathbf{b}(\mathbf{x})$, two independent parameters

$$
p_{1}=\frac{g \cdot \operatorname{si}(\phi)}{l}, \operatorname{si}(\phi)=\frac{\sin \phi}{\phi} \quad \text { and } \quad p_{2}=\frac{\cos (\phi)}{T_{1} \cdot l}
$$

replace the matrix entries depending on the load angle $\phi$, so that the parameter-dependent system matrix and input vector

$$
\mathbf{A}(\mathbf{p})=\left[\begin{array}{cccc}
0 & 0 & 1 & 0 \\
0 & 0 & 0 & 1 \\
0 & 0 & -\frac{1}{T_{1}} & 0 \\
0 & -p_{1} & p_{2} & -d
\end{array}\right] \quad \text { and } \quad \mathbf{b}(\mathbf{p})=\left[\begin{array}{c}
0 \\
0 \\
\frac{1}{T_{1}} \\
-p_{2}
\end{array}\right]
$$

respectively, are obtained. To guarantee the applicability of the presented methods, the controller will be calculated for the worst-case scenarios assuming independent parameters $p_{1}$ and $p_{2}$. The transformation, and hence, the simulation will be done with finer intervals also considering partially dependent parameters. This is still an overapproximation of the real system and therefore offers a secure enclosure of the reality. The controller was calculated for ${ }^{2}$

$$
\begin{aligned}
{[\phi] } & =[-0.1 ; 0.1] \mathrm{rad} \\
{[l] } & =[0.1 ; 0.5] \mathrm{m} \\
{[d] } & =[0.3 ; 1.5] \mathrm{Nm} \cdot \mathrm{s} .
\end{aligned}
$$

Implementing a stability margin of $\gamma=0.1$ and the $\mathrm{H}_{2}$-Norm with $\mathcal{Q}=\operatorname{diag}\left(\left[\begin{array}{llll}1 & 0.1 & 1 & 0.1\end{array}\right]\right)$ and $\mathcal{R}=1$, the controller gains result in

$$
\mathbf{K}=\left[\begin{array}{llll}
0.01 & -0.13 & -0.94 & -0.15
\end{array}\right] .
$$

A further stability proof was done for a widened angle interval of $[\phi]=[-1 ; 1] \mathrm{rad}$. With the smaller intervals representing

\footnotetext{
${ }^{2}$ Note that the boom crane parameters are based on an available test rig at the Chair of Mechatronics which is a scaled model of a real boom crane.
}

the domain of operation as a subset of the stabilized state domain for the system to be transformed

$$
\begin{aligned}
{[\phi] } & =[-0.1 ; 0.1] \mathrm{rad} \\
{[l] } & =[0.4 ; 0.5] \mathrm{m} \\
{[d] } & =[0.5 ; 1.2] \mathrm{Nm} \cdot \mathrm{s},
\end{aligned}
$$

the controlled system matrix than becomes

$$
\mathbf{A}_{\mathrm{C}}=\left[\begin{array}{cccc}
0 & 0 & 1 & 0 \\
0 & 0 & 0 & 1 \\
0.197 & 2.625 & -1.254 & 3.048 \\
a_{41} & a_{42} & a_{43} & a_{44}
\end{array}\right]
$$

with

$$
\begin{aligned}
& a_{41}=[0.392 ; 0.493] \\
& a_{42}=[-31.089 ;-24.812] \\
& a_{43}=[2.495 ; 3.135] \\
& a_{44}=[-8.821 ;-6.565] .
\end{aligned}
$$

The transformation described in Sec. II-B yields to a system matrix

$$
\mathbf{N}=\left[\begin{array}{cccc}
\sigma_{1} & 0 & 0 & 0 \\
0 & \sigma_{2} & 0 & 0 \\
0 & 0 & \sigma_{3} & 0 \\
0 & 0 & 0 & \sigma_{4}
\end{array}\right]
$$

which is clearly Metzler and Hurwitz with

$$
\begin{aligned}
\sigma_{1}=\sigma_{2} & =[-4.321 ;-3.141] \\
\sigma_{3} & =[-1.242 ;-1.220] \\
\sigma_{4} & =[-0.194 ;-0.192] .
\end{aligned}
$$

Note that, the real parts of the first two eigenvalues $\sigma_{1}$ and $\sigma_{2}$ are equal since they belong to a conjugate-complex pair.

\section{Simulation RESUlts}

Now, we will have a closer look into subdividing possibilities, see Sec. II. As mentioned in the previous section, a parameter independency with additional pessimism is introduced by choosing $p_{1}$ and $p_{2}$. Hence, our system (42) has (a) three independent parameters: $p_{1}, p_{2}$ and $d$. However, when subdividing $p_{1}$ and $p_{2}$ independently, we ignore the fact, that both still depend on the same length $l$. This can be accounted for, if (b) we choose to grid $l$, then calculate the parameters and finally grid them again. It is then obvious to (c) introduce a further subdivision of the parameter $\phi$, omitting the subdivision of $p_{1}$ and $p_{2}$ and hence, reduce the effort by one subdivision. Fig. 3 presents how the positions of eigenvalues vary for the different approaches, showing the least conservative to be the subdivision of $\phi, l$ and $d$. Hence, (c) is the approach used to calculate the interval enclosures. Since this subdivision resembles $L^{3}$ small subintervals, it becomes important to know which hull is preferable to consider, see Sec. II. All three methods are shown, exemplary for the angle $\phi$, in Fig. 4 for the uncertain initial states

$$
\mathbf{x}(0)=\left[\begin{array}{c}
{[0.1 ; 0.2] \mathrm{m}} \\
{[-0.1 ; 0.1] \mathrm{rad}} \\
{[0.01 ; 0.02] \mathrm{m} \cdot \mathrm{s}^{-1}} \\
{[0.05 ; 0.08] \mathrm{rad} \cdot \mathrm{s}^{-1}}
\end{array}\right]
$$

As one can see, there is not much difference in the resulting interval enclosures. However, Approach (3) (- solid line) is 


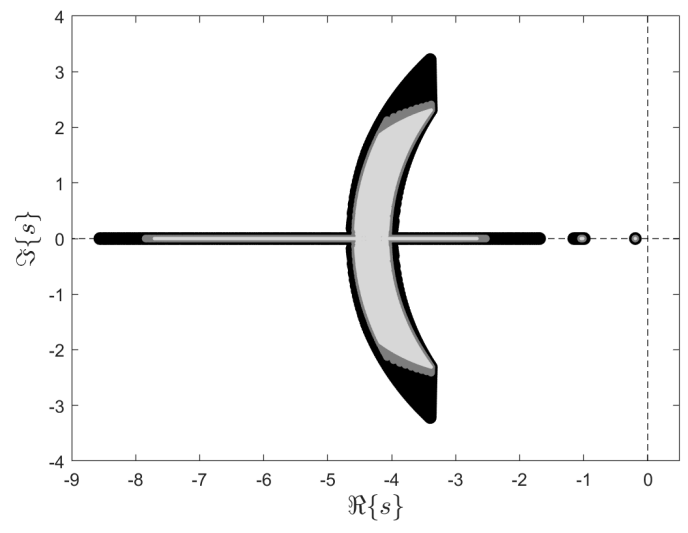

Figure 3. Distribution of eigenvalues: (a) subdivision of $p_{1}, p_{2}$ and $d-$ black, (b) subdivision of $l, p_{1}, p_{2}$ and $d$ - dark gray and (c) subdivision of $\phi, l$, and $d-$ light gray.

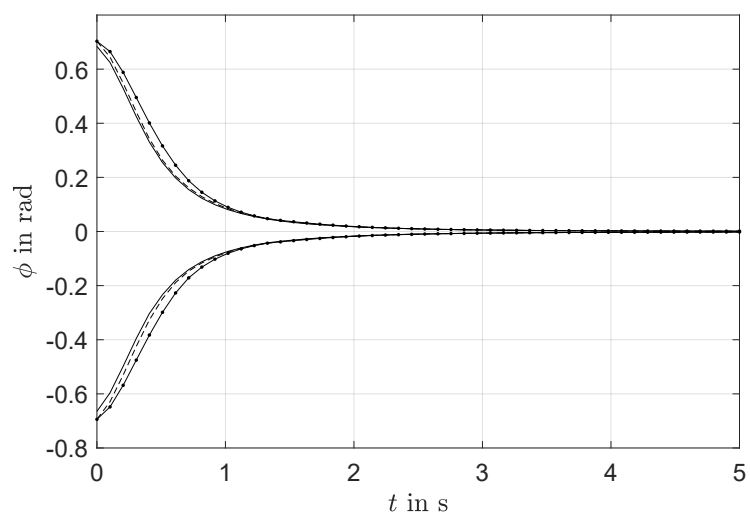

Figure 4. Interval enclosure for the state $\phi-$ approaches from II-B: 1. $\tilde{\mathbf{T}}=\left[\tilde{\mathbf{T}}_{1}, \ldots, \tilde{\mathbf{T}}_{\tilde{n}}\right]$ with $\tilde{\mathbf{T}}_{j} \in\left[\bigcup_{\kappa=1}^{L}\left[\Re\left\{\left[\mathbf{v}_{j}\right]\right\}^{i}\right], \bigcup_{\kappa=1}^{L}\left[\Im\left\{\left[\mathbf{v}_{j}\right]\right\}^{i}\right]\right]-$ dotted, 2. $[\tilde{\mathbf{T}}]=\bigcup_{\kappa=1}^{L}\left[\tilde{\mathbf{T}}^{i}\right]$ - dashed, 3. $[\mathbf{z}]=\bigcup_{\kappa=1}^{L}\left[\mathbf{z}^{i}\right]$ - solid.

the least conservative, which is due to the subdistributivity of interval analysis [3]. Furthermore, the inverse transformation matrix can be difficult to compute if wide intervals are included, making Approach (1) impossible to apply. Hence, it is recommendable to calculate the inverse transformation matrix for each subinterval and build the hull over those.

\section{CONCLUSion}

The oscillation of the load of a boom crane was damped by a robust control based on an LMI approach considering $\Gamma$-regions for user defined stability regions and an $\mathrm{H}_{2}$-norm optimization for the limitation of the control effort. The guaranteed state enclosures of the controlled system were computed after a transformation to exploit the property of cooperativity. Those enclosures serve as a prerequisite for a guaranteed stabilizing control. Here, an analysis was given on how to choose methods of subdivision to suitably calculate the results avoiding overestimation that does not reflect reality due to lumping the uncertainty into independent parameters. In future work, these calculated interval enclosures can be used to optimize gain scheduling controllers comparable with the work in [10]. Additionally, cooperativity preserving observers, see [19] can be implemented and, finally, an experimental validation on the test rig of the Chair of Mechatronics will take place.

\section{REFERENCES}

[1] A. Rauh, E. Auer, and E. P. Hofer, "VALEnCIA-IVP: A Comparison with Other Initial Value Problem Solvers," in CD-Proc. of the 12th GAMM-IMACS Intl. Symposium on Scientific Computing, Computer Arithmetic, and Validated Numerics SCAN 2006. Duisburg, Germany: IEEE Computer Society, 2007.

[2] N. S. Nedialkov, "Interval Tools for ODEs and DAEs," in CD-Proc. of the 12th GAMM-IMACS Intl. Symposium on Scientific Computing, Computer Arithmetic, and Validated Numerics SCAN 2006. Duisburg, Germany: IEEE Computer Society, 2007.

[3] L. Jaulin, M. Kieffer, O. Didrit, and É. Walter, Applied Interval Analysis. London: Springer-Verlag, 2001.

[4] W. Kühn, "Rigorous Error Bounds for the Initial Value Problem Based on Defect Estimation," Tech. Rep., 1999, available online: http://www. decatur.de/personal/papers/defect.zip.

[5] V. Puig, J. Saludes, and J. Quevedo, "Worst-Case Simulation of Discrete Linear Time-Invariant Interval Dynamic Systems," Reliable Computing, vol. 9, no. 4, pp. 251-290, 2003.

[6] T. Kaczorek, Positive $1 D$ and $2 D$ Systems. London: Springer-Verlag, 2002.

[7] H. L. Smith, Monotone Dynamical Systems: An Introduction to the Theory of Competitive and Cooperative Systems. Mathematical Surveys and Monographs, American Mathematical Soc., 1995, vol. 41.

[8] J. Kersten, A. Rauh, and H. Aschemann, "State-Space Transformations of Uncertain Systems With Purely Real and Conjugate-Complex Eigenvalues Into a Cooperative Form," in Proc. of 23rd Intl. Conf. on Methods and Models in Automation and Robotics 2018, Miedzyzdroje, Poland, 2018.

[9] T. Raïssi, D. Efimov, and A. Zolghadri, "Interval State Estimation for a Class of Nonlinear Systems," IEEE Trans. Automat. Contr., vol. 57, pp. 260-265, 2012.

[10] J. Kersten, A. Rauh, and H. Aschemann, "Application-Based Discussion of Verified Simulations of Interval Enclosure Techniques," in Proc. of 24th International Conference on Methods and Models in Automation and Robotics 2019, Miedzyzdroje, Poland, 2019.

[11] _ , "Interval Methods for the Implementation and Verification of Robust Gain Scheduling Controllers," in Proc. of 22nd Intl. Conf. on Methods and Models in Automation and Robotics 2017, Miedzyzdroje, Poland, 2017.

[12] _ - "Interval methods for robust gain scheduling controllers - An LMI-based approach," Granular Computing, pp. 203-216, 2018.

[13] C. Scherer and S. Weiland, "Linear Matrix Inequalities in Control," in Control System Advanced Methods, 2nd ed., ser. The Electrical Engineering Handbook Series, W. S. Levine, Ed. Boca Raton: CRC Press, 2011, pp. 24-1-24-30.

[14] J. Ackermann, Robust Control - The Parameter Space Approach. Springer-Verlag, 2002.

[15] J. F. Sturm, "Using SeDuMi 1.02, A MATLAB Toolbox for Optimization over Symmetric Cones," Optimization Methods and Software, vol. 11-12, no. 1-4, pp. 625-653, 1999.

[16] J. Löfberg, "YALMIP: A Toolbox for Modeling and Optimization in MATLAB," in Proceedings of IEEE Intl. Symposium on Computer Aided Control Systems Design, Taipei, Taiwan, 2004, pp. 284-289.

[17] T. Raïssi and D. Efimov, "Some Recent Results on the Design and Implementation of Interval Observers for Uncertain Systems," atAutomatisierungstechnik, vol. 66, no. 3, pp. 213-224, 2018.

[18] F. Mazenc and O. Bernard, "Asymptotically Stable Interval Observers for Planar Systems With Complex Poles," IEEE Transactions on Automatic Control, vol. 55, no. 2, pp. 523-527, 2010.

[19] A. Rauh, J. Kersten, and H. Aschemann, "Techniques for Verified Reachability Analysis of Quasi-Linear Continuous-Time Systems," in Proc. of 24th Intl. Conf. on Methods and Models in Automation and Robotics 2019, Miedzyzdroje, Poland, 2019.

[20] S. Rump, "INTLAB - INTerval LABoratory," in Developments in Reliable Computing, T. Csendes, Ed. Kluver Academic Publishers, 1999, pp. 77-104. 